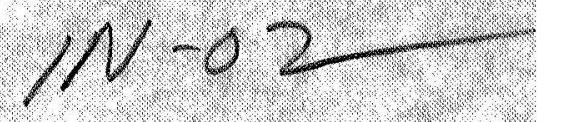

NASA Technical Memorandum 106235

AlAA-93-3253

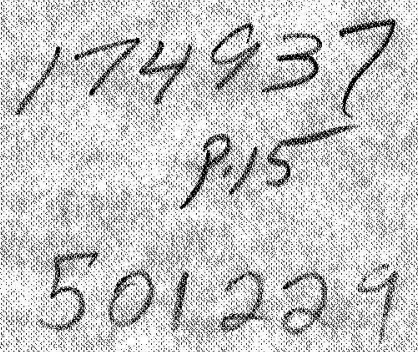

\title{
Streamwise Vorticity Generation and Mixing Enhancement in Free Jets by 'Delta-Tabs'
}

K.B.M.Q. Zaman

Lewis Research Center

Cleveland, Ohio

\section{Prepared for the}

AIAA Shear Flow Conference

sponsored by the American Institute of Aeronautics and Astronautics

Orlando, Florida, July 6-9, 1993



Unclas 


\title{
STREAMWISE VORTICITY GENERATION AND MIXING ENHANCEMENT IN FREE JETS BY 'DELTA-TABS'
}

\author{
by
}

\author{
K. B. M. Q. Zaman \\ NASA Lewis Research Center \\ Cleveland, OH 44135
}

\begin{abstract}
The effect of triangular tabs, placed at the nozzle exit, on the evolution of free jets is investigated. The effect, a large distortion of the jet cross section and a resultant increase in mixing downstream, has been inferred before to be due to a pair of streamwise vortices originating from each tab. In this paper, the generation mechanism of the streamwise vorticity $\left(\omega_{x}\right)$ is considered first. Two sources are postulated. One is the upstream 'pressure hill', produced by the tab, which appears to be the dominant source. Another is due to vortex filaments shed from the sides of the tab and reoriented downstream by the mean shear of the mixing layer. In the case of a 'delta-tab', a triangular tab with its apex leaning downstream, vorticity from the two sources add explaining the stronger effect in that configuration. Data on the vorticity evolution for the effect of two delta-tabs are presented, up to twelve jet diameters from the exit, which show that the streamwise vortices persist even at the farthest measurement station. The magnitude of $\omega_{x}$-maximum decays continually with distance from the nozzle, its ratio to azimuthal vorticity $\left(\omega_{\theta}\right)$ maximum is found to be about 1/5 everywhere. The relative effect of a delta-tab on jets from an axisymmetric nozzle and a 8:1 rectangular nozzle is also studied. The mixing layer distortion is found to be less pronounced in the rectangular case. The jet mixing, as manifested by the mass flux measured at a downstream station, is increased in the axisymmetric jet but it is decreased in the rectangular jet under consideration by the delta-tab.
\end{abstract}

\section{Introduction}

Results of a continuing experimental investigation on the effect of vortex generators, in the form of 'tabs' placed at the nozzle exit, on the evolution of free jets are presented in this paper. The effect of triangular tabs with apex leaning downstream, referred to as 'delta-tabs', is mainly considered. The motivation of the study stems from the need for enhancing mixing especially in supersonic shear flows encountered in a variety of technological applications.

Previous results of the investigation on the effect of simple rectangular tabs as well as 'delta-tabs' have been presented in Refs. 1 and 2. Based on flow visualization results, it was conjectured that each tab produced a pair of streamwise vortices with a sense of rotation opposite to that of 'necklace' vortices commonly occurring in flows over protuberances in boundary layers. The generation mechanism of the streamwise vorticity in the tabbed jet case has been considered recently. ${ }^{3}$ In the present paper, this is discussed first with additional details. Two sources of the vorticity are postulated based on the pressure gradients produced by the tab. The postulated sources enable a rational explanation for several observations made with the tabs.

In the earlier experiments, ${ }^{3}$ only limited hot-wire measurements could be conducted to qualitatively demonstrate the presence of the streamwise vortices. These measurements were done in a smaller diameter jet where probe resolution was a serious problem. It was felt that further measurements were called for, while alleviating this problem, not only to confirm the presence of the vortices but also to quantify them so that the knowledge of this technologically important flow could be advanced. Detailed measurements of the flow field were carried out in a larger diameter jet with and without two diametrically placed delta-tabs. The corresponding evolution of vorticity and turbulence statistics are discussed next in the paper.

Another issue addressed is the comparative effect of a delta-tab in axisymmetric versus rectangular nozzle configurations. This investigation was prompted by prior studies (Ref. 4) which suggested a diminished effect of a tab in a rectangular jet. Limited measurements were 
carried out with a 8:1 rectangular jet. The results do indicate a reduced but complex effect of the delta-tab in this jet as compared to the effect in the axisymmetric jet. An attempt is made to explain the difference in the initial evolution of the flow in the two configurations based on the vorticity generation mechanism.

\section{Experimental Facility and Method}

The experiments were carried out in two different jet facilities at the NASA Lewis Research Center. Facility \#1 was the same as used for the experiments reported in Refs. 1-3. Compressed air supplied through one end of a $11 \mathrm{~cm}$ diameter plenum chamber exited through a $3.81 \mathrm{~cm}$ diameter 'nipple' on the opposite end of the plenum. Nozzles of different exit geometries could be attached to the nipple. The supersonic jet experiments were performed in this facility. Facility \#2 was a larger one with a $76 \mathrm{~cm}$ diameter plenum and was described, e.g., in Ref. 5. A $8.9 \mathrm{~cm}$ diameter nozzle, also described in Ref. 5 , was used with this facility for the measurement of vorticity evolution in a subsonic jet. All nozzles used in the investigation were convergent ones.

A triangular tab fitted to a circular nozzle is shown schematically in Fig. 1. The specific configuration with orientation $\phi-135^{\circ}$ is referred to as the 'delta-tab'. The apex angle is about $90^{\circ}$, and the dimensions are such that the blockage due to each delta-tab is about 1.5 percent of the nozzle exit area (w/D -0.28$)$. The delta-tabs were hand sheared and bent and thus the angles and dimensions quoted are approximates.
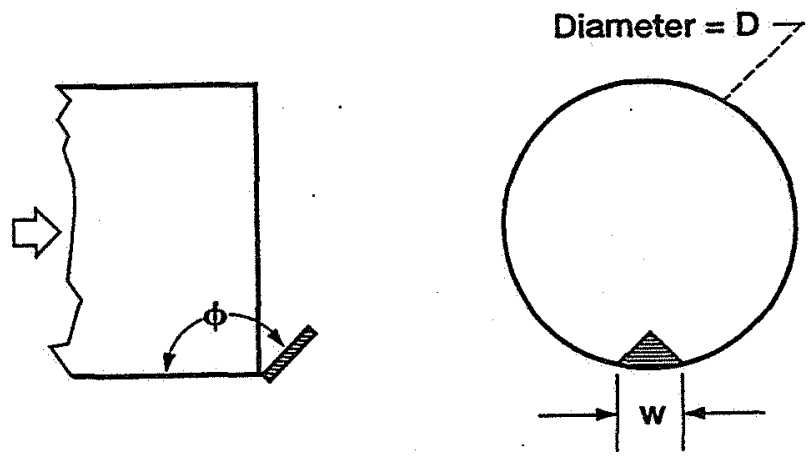

Fig. 1 Schematic of nozzle end with triangular tab.
For the supersonic jets, the notation $M_{j}$ is used to denote the 'fully expanded Mach number', i.e., the Mach number had the jet expanded to ambient pressure $\left(p_{2}\right)$ for a given plenum pressure, $p_{10}$. The notation $P$ represents static pressure. Standard Pitot tube measurements were conducted to obtain variations of the stagnation pressure $\left(p_{1}\right)$, and distributions of Mach number in the subsonic regions of the flow.

Flow visualization pictures for the supersonic jets were obtained by laser sheet illumination. This was done without any artificial seeding of the flow. The cold supersonic jet core caused natural moisture condensation in the mixing layer. The condensed moisture particles, and therefore the mixing layer regions, were illuminated by the laser sheet. The exposure time $(T)$ for each picture was long $\left(\mathrm{TU}_{\mathrm{j}} / \mathrm{D}>270\right)$ so that these represent averages rather than instantaneous flow fields.

In the larger jet facility, conventional hot-wire measurements were done to obtain distributions of time averaged vorticity and turbulence statistics on a cross sectional plane of the jet. The measurements could be carried out only for the subsonic flow, and $M_{j}=0.3$ was chosen. Two X-wire probes, one in the u-v and the other in the $u$-w configuration, were traversed successively over the same measurement grid locations. The distributions of the $u, v$ and $w$ components of velocity were obtained over the measurement plane. The gradients of $v$ and $w$ provided the streamwise vorticity. These measurements were done in the larger jet so that the spatial resolution provided by the (TSI 1241) X-probes was reasonably good. Nevertheless, simple estimates, based on mixing layer thickness and sensor separation in the X-probe $(-1 \mathrm{~mm})$, showed that corrections were needed for the $\mathrm{v}$ - and $\mathrm{w}$ components of velocity especially at the upstream locations. The data were corrected following a scheme similar to that used in Ref. 6.

\section{Results}

3.1 Vorticity generation mechanism: The effect of the tabs on the jet cross section was most vividly illustrated by the laser sheet flow visualization. For a supersonic jet the effect of one triangular tab, with different orientation $\phi$ to the stream direction, is shown in Fig. 2. The general effect is to produce a large indentation into the core of the jet. Further details of the effect of multiple triangular tabs compared to the no-tab case can be found in Ref. 2 . The 


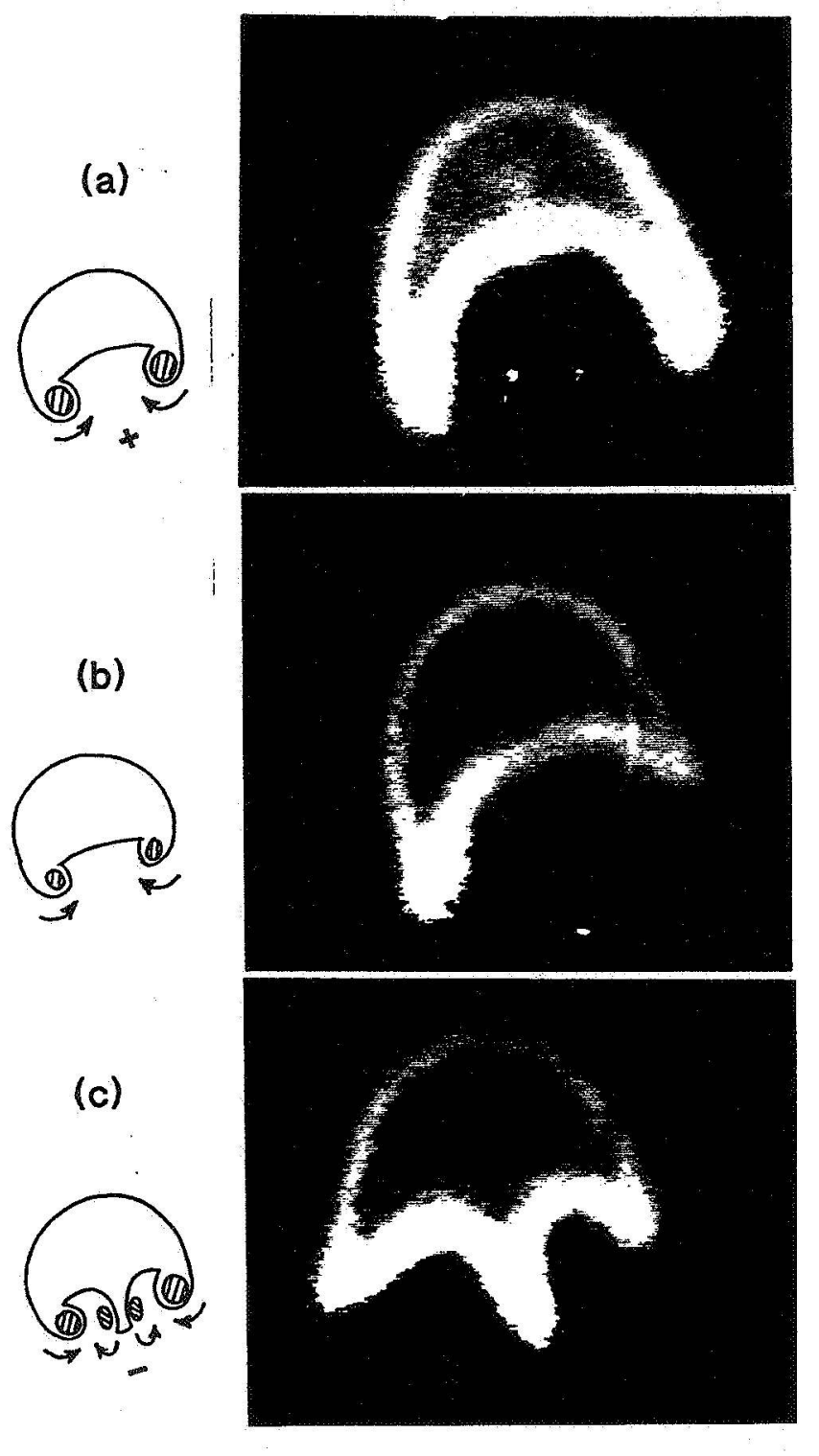

Fig. 2 Laser sheet illuminated cross section of jet at $\mathrm{x} / \mathrm{D}=2 ; \mathrm{D}=1.27 \mathrm{~cm}, \mathrm{M}_{\mathrm{j}}=1.63$. Triangular tab with: (a) $\phi=135^{\circ}$, (b) $\phi=90^{\circ}$, (c) $\phi=45^{\circ}$.

purpose here is to compare the relative effect of the tab when the orientation is varied. One observes that the indentation for $\phi=135^{\circ}$ (delta-tab) is more pronounced than that for $\phi=90^{\circ}$. The corresponding picture for $\phi$ $=45^{\circ}$ clearly shows an outward bulge, or core fluid ejection, in the middle of the indentation.
The likely vorticity distributions on the cross sectional plane for the three cases of Fig. 2 are shown by the sketches on the side of each picture. The distributions are similar in Figs. 2(a) and (b) except that the indentation, and therefore the streamwise vortices, are more pronounced in (a). The sense of rotation of these vortices are opposite to what would be expected from the wrapping of the upstream boundary layer. For the purposes of the following discussion the pair of vortices in (a) or (b) will be called to have a positive sense of rotation. The outward bulge in Fig. 2(c) is believed to be caused by a pair of vortices having a negative sense of rotation and this is represented by the inner pair in the corresponding sketch. However, there is an overall indentation in this case not unlike that observed for the $90^{\circ}$ and $135^{\circ}$ cases, and this should be due to a second outer pair with a positive sense of rotation as shown in the sketch. These vorticity distributions are explained by the mechanism postulated later in this section.

The pressure gradients created by the tabs, together with the presence of the solid surfaces, are thought to be the main sources of streamwise vorticity in the present flow. The static pressure distributions upstream of the tab were measured. These measurements were performed within the cylindrical section of the nozzle. Data for $M_{j}$ $=1.63$ are shown in Fig. 3. The streamwise distribution upstream of the tab is shown in (a); $\mathrm{x}=0$ denotes the tab location at the nozzle exit. Data for $\phi=135^{\circ}$ and $45^{\circ}$ are compared with the data for the no-tab case. As expected, the maximum static pressure is produced just upstream of the tab; the amplitude is slightly more for the $\phi=45^{\circ}$ case. The amplitude diminishes with increasing distance away from the tab. The corresponding azimuthal distributions of static pressure at $\mathrm{x} / \mathrm{D}=-0.2$ are shown in Fig. 3(b); $\psi=0$ denotes the tab location. The small azimuthal variation of the amplitude for the no-tab case might represent standing waves due to disturbances of certain azimuthal modes; however, this is considered unimportant in the present discussion. One finds that the peak in the static pressure occurs just upstream of the tab(s) and the magnitude falls off gradually on either side.

Also shown in Fig. 3 are the corresponding static pressure variations when a tab is placed with a gap between the nozzle exit and itself. The gap effect is brought up here because it delineates the linkage between the pressure distributions and the jet distortion produced via the streamwise vortices. It can be seen that with the 

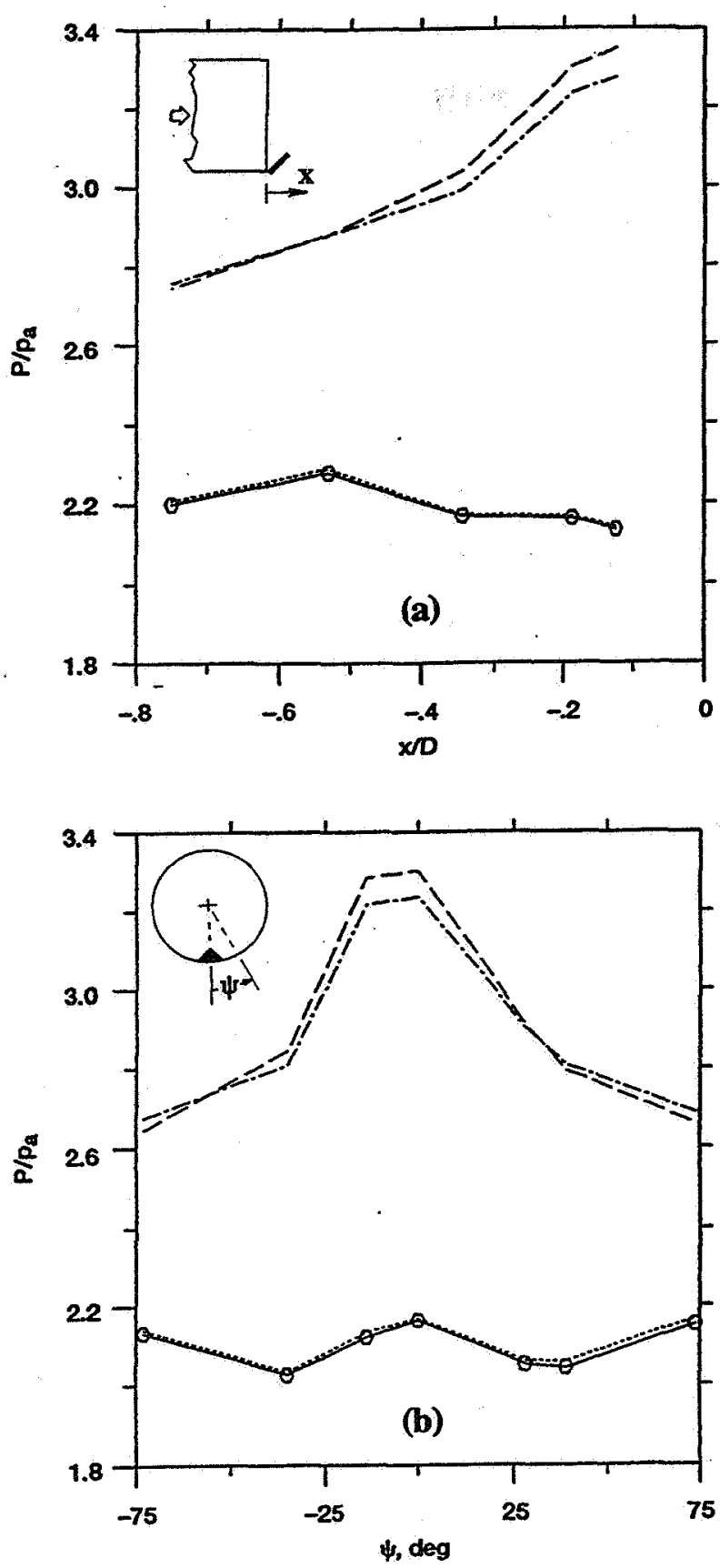

Fig. 3 Static pressure distributions in the cylindrical section of $1.27 \mathrm{~cm}$ diameter nozzle, $M_{j}=1.63$.

(a) streamwise distribution $\left(\psi=0^{\circ}\right)$; (b) aximuthal distribution $(x / D=-0.2)$. $\ldots \ldots$, triangular tab $\phi=45^{\circ} ; \ldots \ldots . \ldots$, , triangular tab $\phi=135^{\circ}$ (delta-tab); . ........., rectangular tab with gap equal to width.

gap the resultant pressure distribution is just about the same as that in the no-tab case. Flow visualization results reported in Ref. 1 showed that the gap also resulted in a complete disappearance of the jet distortion which was otherwise produced by the tab; this was true for both supersonic and subsonic jets. The data shown in Fig. 3 on the gap effect are for a rectangular tab; a cursory check with delta-tabs also showed a similar result. Water tunnel flow visualization recently reported in Ref. 7 confirmed that the effect of the delta-tab diminished when placed with a gap. The gap effect leads to further inferences regarding the vorticity generation mechanism as explained in the following.

A more detailed distribution of the upstream static pressure, obtained in connection with the data to be presented in $\$ 3.2$, is shown in Fig. 4. These data are for the effect of a delta-tab in the larger jet at $M_{j}=0.3$. The data of Figs. 3 and 4 demonstrate that each tab, when placed against the nozzle lip, sets up a 'pressure hill' just upstream of itself. The peak amplitude of this 'pressure hill' gradually falls off with increasing distance from the tab.

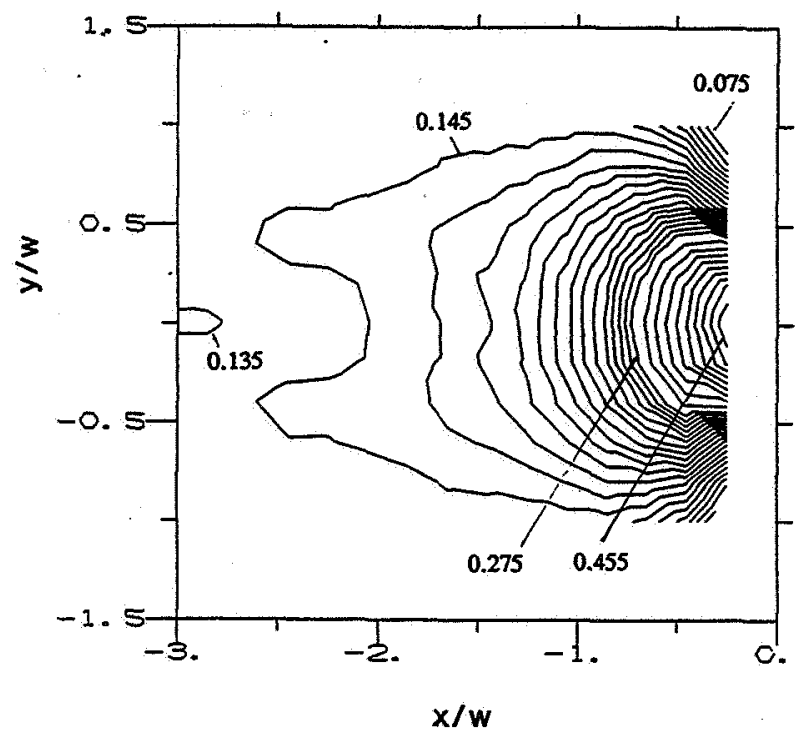

Fig. 4 Contours of static pressure, $P /\left(1 / 2 \rho U_{j}^{2}\right)$, upstream of delta-tab and near nozale wall; $D=8.9$ $\mathrm{cm}, M_{j}=0.3$. Contour intervals are 0.01 and 0.02 below and above the level 0.275 , respectively.

It should be emphasized here that the overall effect of the tab is essentially similar at supersonic and subsonic flow regimes. In addition to the similar effect of the gap 
discussed in the foregoing, vortex interactions in flows with multiple delta-tabs was also found to be identical in the two flow regimes. ${ }^{3}$ There are additional effects of the tab on the shock train and the screech in supersonic jets, but these effects are considered secondary. The primary effect is the generation of the streamwise vortices which is essentially the same at all $\mathrm{M}_{\mathbf{j}}$.

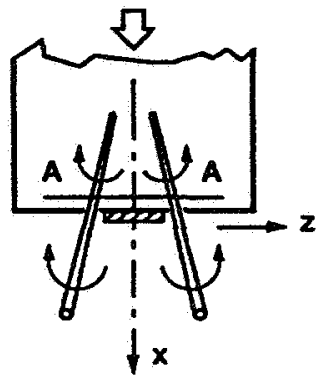

(a)

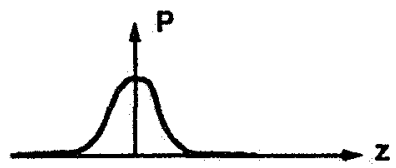

(c)

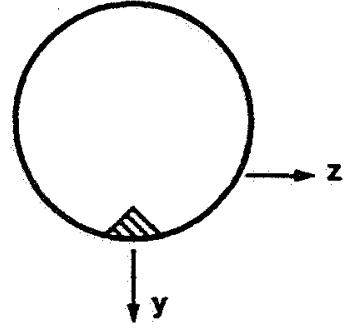

(b)

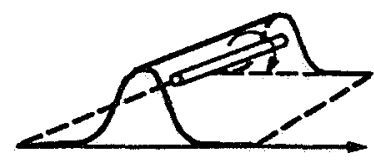

(d)
Fig. 5 'Pressure hill' upstream of tab as source \#1 for generation of streamwise vorticity.

Two sources of streamwise vorticity may be recognized in the flow under consideration. "Source 1 is the upstream 'pressure hill', as elaborated schematically in Fig. 5. The top and the end views of the nozzle with the tab are shown in Fig. 5(a) and (b), respectively. The pressure gradient in the $z$-direction (c), together with the presence of the nozzle wall, would produce a pair of streamwise vortices as shown in (a). The vorticity flux produced by

The possibility of the two sources of streamwise vorticity was originally suggested by Professor J.F. Foss of Michigan State University; the author gratefully acknowledges his help in the development of the ideas presented in \$3.1. the pressure gradient in this case, assuming incompressible flow, is given by, ${ }^{8}$

$$
\frac{1}{\rho} \frac{\partial p}{\partial z}=v \frac{\partial \omega_{x}}{\partial y}
$$

It is thought that other vorticity sources which are operative in compressible flows, e.g. unsteady flow interaction with shock, 'baroclinic torque', etc., ${ }^{9}$ must not be significant, simply because a similar overall effect of the tab is observed in supersonic and subsonic conditions. The pair of vortices produced would have the 'positive' sense of rotation; (the vortices could be thought of as 'rollers' rolling down the sides of the 'pressure hill', as sketched in Fig. 5d). It should be emphasized that all data discussed in this paper are steady flow results based on time averages. The 'pressure hill' is also a steady source producing a steady pair of streamwise vortices.

Vorticity source 2 is sketched in Fig. 6. Sheets of vorticity are shed from the sides of the tab (Fig. $6 \mathrm{c}$ ). This is also a steady source and can again be attributed to the pressure distributions produced by the tab (Fig. 6d). In Fig. 6(d), the pressure is shown to be slightly lower than ambient pressure at the edges which may be expected due to streamline curvature; but this is unimportant in the present context. A vortex filament, which is a part of the vortex sheet shed over a finite time, is initially aligned with the side of the delta-tab from which it is shed (Fig. 6c). But as it propagates downstream it is reoriented by the velocity gradient, $\partial u / \partial y$. In the case of the vortex filaments for the actual delta-tab case, the components $\omega_{y}$ and $\omega_{z}$ would be reoriented to produce $\omega_{x}$ via the terms $\omega_{y} \partial u / \partial y$ and $\omega_{z} \partial u / \partial z$ in the vorticity transport equation. ${ }^{10,11}$

One can now see that the reorientation is accomplished with relative ease in the delta-tab case as the filament has already started with a favorable inclination (Fig. 6e). For the triangular tab with $\phi=45^{\circ}$ the reorientation may be accomplished only partially for the part of the filament lying in the mixing layer (Fig. 6f). Most of the filament, contained in the jet core, would retain the original orientation yielding a vortex pair with 'negative' sense of rotation. It is, however, clear that streamwise vorticity from sources 1 and 2 will add in the delta-tab case, both contributing to the formation of the 'positive' vortex pair. This should explain the stronger effect in the latter case. 




(a)

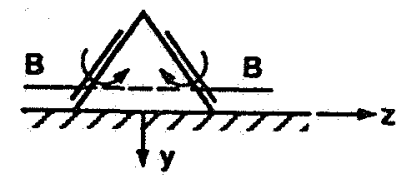

(c)

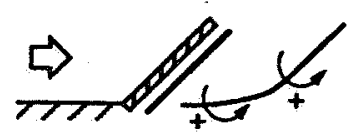

(e)

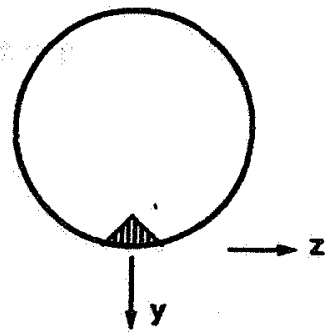

(b)

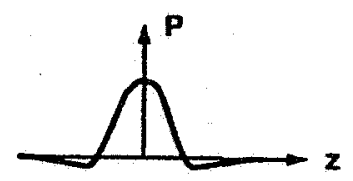

(d)

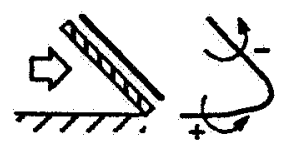

(0)
Fig. 6 Source $\$ 2$ of streamwise vorticity. (a), (b) views of nozzle with tab; (c) end view of tab showing two vortex filaments; (d) pressure distribution at B$B$; (e) for delta-tab reorientation of near side vortex filament; (f) for $\phi=45^{\circ}$ case, vortex filament only partially reoriented.

It should also take a minor extension of the same reasoning to explain the stronger effect of the delta-tab compared to the case when the tab is placed with $\phi=90^{\circ}$.

For the tab with $\phi=45^{\circ}$, one would expect a 'positive' vortex pair originating mainly from source 1 which is partially augmented by source 2 . In addition, a second 'negative' vortex pair from source 2 is also expected. This reasoning led to the sketch in Fig. 2(c). When the tab is placed with a gap, the pressure gradients diminish partially or completely (Fig. 3), thus removing or diminishing source 1 . Even though a diminished pressure gradient would also be expected to diminish the strength of source 2, the latter source should still be operative when the tab is placed with a gap. Vortex filaments should be shed from the sides of the tab and reoriented downstream as sketched in Fig. 6. Thus, the ineffectiveness of the tab in the case with the gap suggest that source 1 is the dominant one for the generation of streamwise vorticity in the present flow.
3.2 Flow field for the effect of 2 delta-tabs at $M_{\mathrm{i}}=0.3$ : Detailed flow field measurements were carried out for the effect of two diametrically placed delta-tabs. Besides attempting to shed further light onto the flow mechanisms these measurements were made to also provide a data base for ongoing computational studies of the subject. ${ }^{12}$ Only limited tab cases could be explored, however, in view of the time consuming nature of these measurements. The case of 2 delta-tabs was chosen because the vorticity evolution downstream leading to the 'bifurcation' of the jet was considered interesting. ${ }^{3}$ The static pressure distribution upstream of one of the two delta-tabs has been discussed in the preceding section (Fig. 4). Other key results from this set of experiments are documented and briefly discussed in the following.

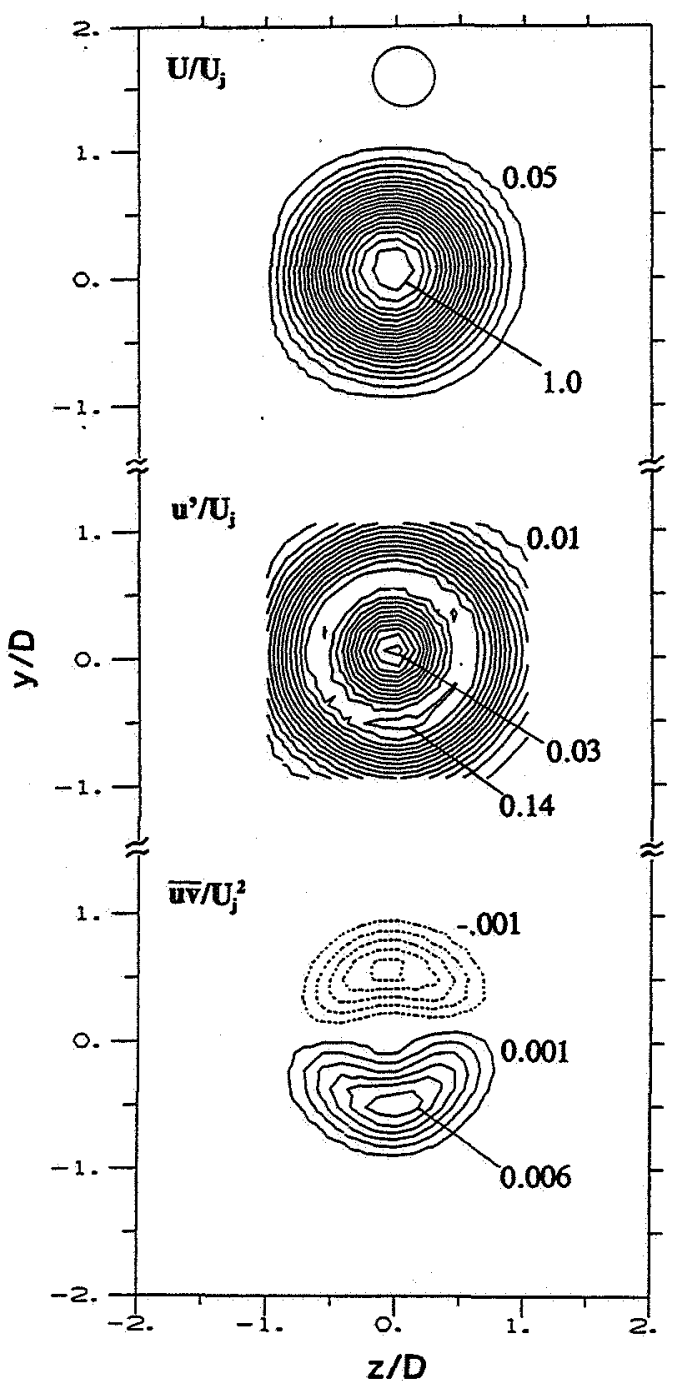

Fig. 7 Contours of indicated quantities for the no-tab case; $x / D=3, M_{j}=0.3$. 
The mean velocity (U), longitudinal turbulence intensity ( $\left.u^{\prime}\right)$ and Reynolds stress (ūv) distributions, measured at $x / D=3$ for the no-tab case, are first shown in Fig. 7. Corresponding data for the 2 delta-tabs case are shown in Fig. 8. The $U$ and $u^{\prime}$ data demonstrate the significant effect of the delta-tabs. The circular crosssection of the jet has been distorted and squeezed out into two cores. The increase in the area of the U-contours indicate an increase in the volume flux and hence in the mixing. Peak values of the turbulence intensity as well as the Reynolds stress have also increased substantially.

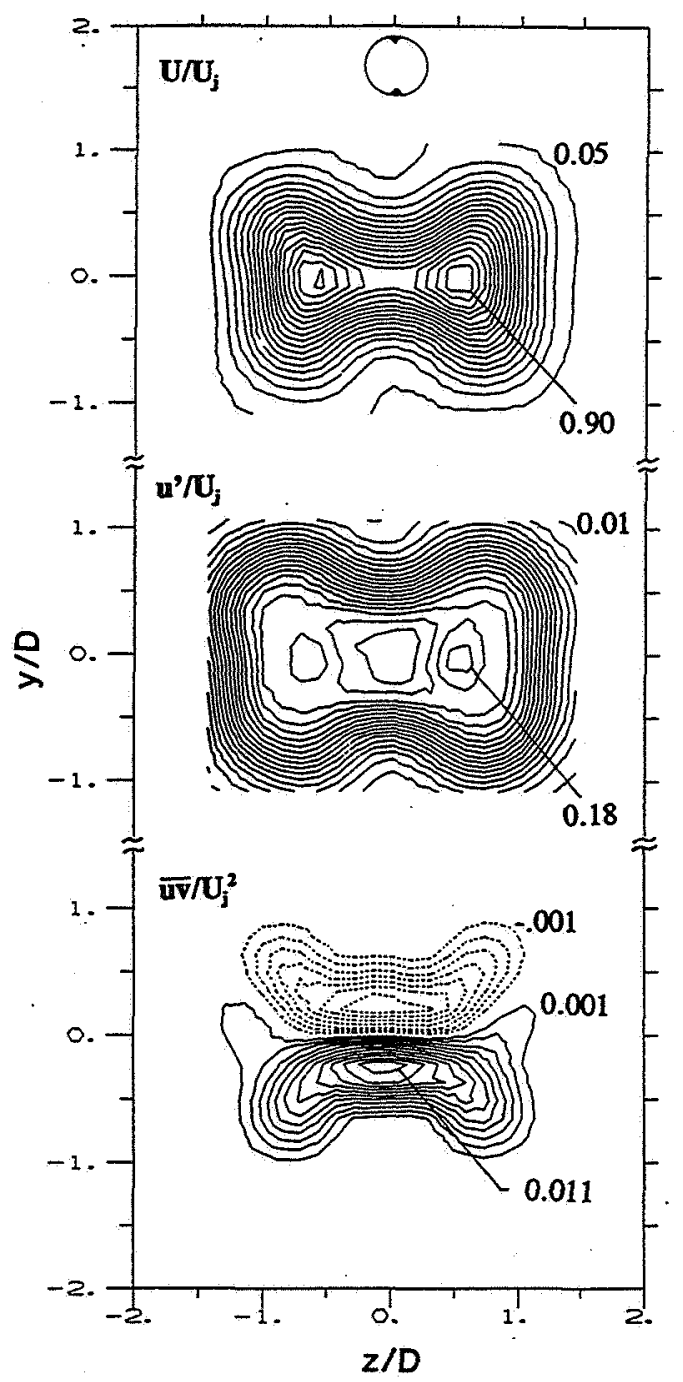

Fig. 8 Contours of indicated quantities for 2 deltatabs case; $x / D=3, M_{j}=0.3$.
The mean velocity distributions along the jet centerline are shown in Fig. 9; also shown in this figure for the tab case are the values of the U-maxima measured at each $x$-station. The data were obtained by keeping the nozzle pressure ratio the same. Thus the velocities at the exit plane were nearly identical for the tab and the no-tab cases. Immediately downstream of the nozzle an increase in the mean velocity is observed most likely due to the acceleration of the flow caused by the delta-tabs. Farther downstream, the velocities $U$ on the centerline, and even the U-maxima, are found to decay much faster under the influence of the delta-tabs. ${ }^{13,14}$

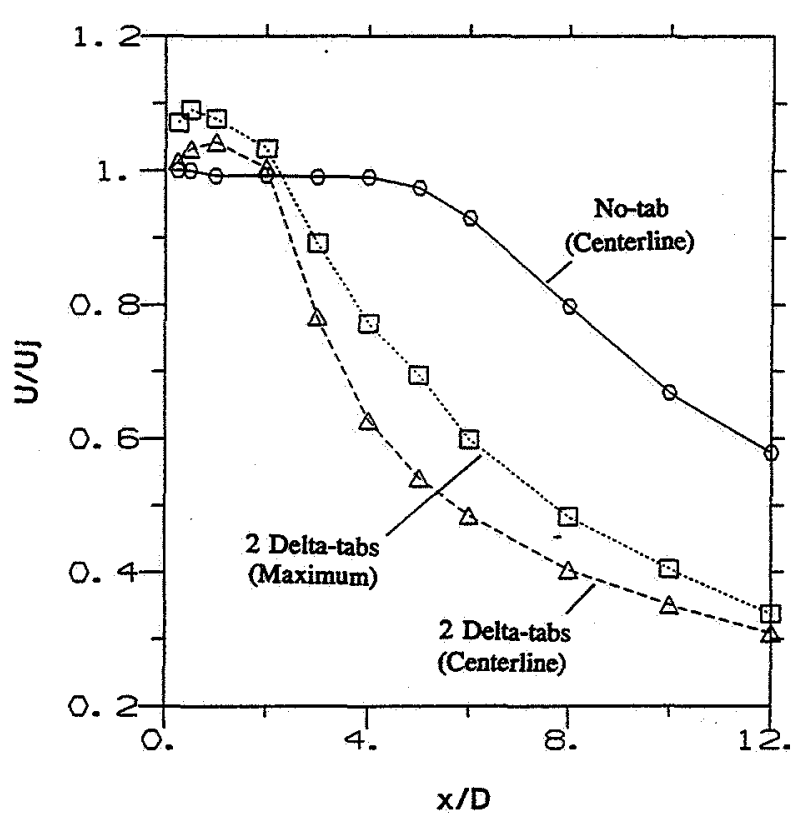

Fig. 9 Streamwise variations of mean velocity for indicated cases.

The maximum values of the turbulent stresses measured at different $x / D$ are shown in Figs. 10 and 11. (The stress $\overline{\mathrm{vw}}$ could not be measured because $\mathrm{v}$ and $\mathrm{w}$ were obtained by two different probes; \$2). Detailed cross sectional distributions for the no-tab case were obtained only for $x / D=3$ and 12 ; the corresponding stress maxima are shown by the solid data points. The levels for the tab case are lower at $x / D=12$ because the jet velocities have decayed considerably by that distance. The levels at $x / D=3$ should be representative of the highest values of the stresses in the entire flow for the no-tab 
case. ${ }^{15}$ Thus, one observes that all the stress maxima have increased substantially under the influence of the tabs.

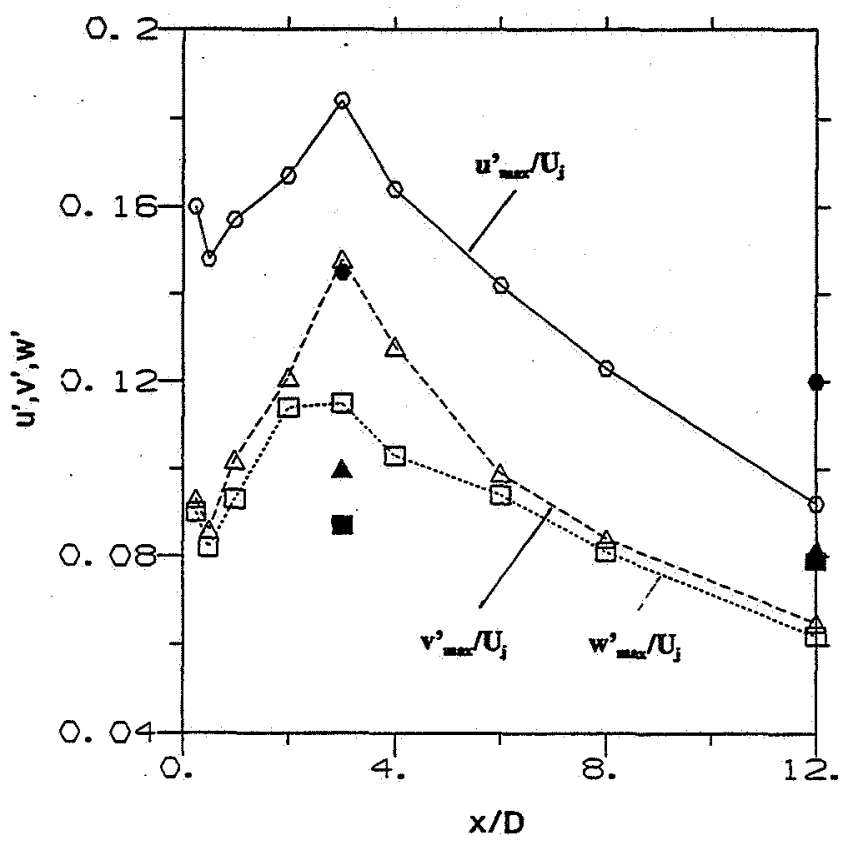

Fig. 10 Streamwise variations of maximum normal stresses for the 2 delta-tabs case; solid data points at $x / D=3$ and 12 are for the corresponding no-tab case.

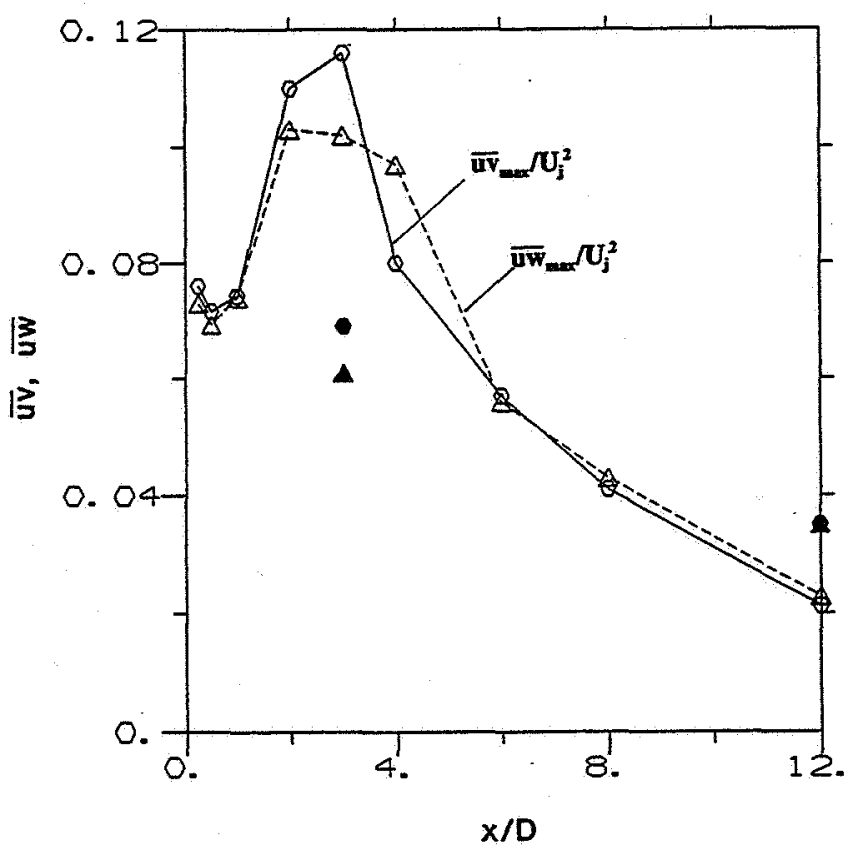

Fig. 11 Streamwise variations of maximum shear stresses for the 2 delta-tabs case; solid data for notab case.

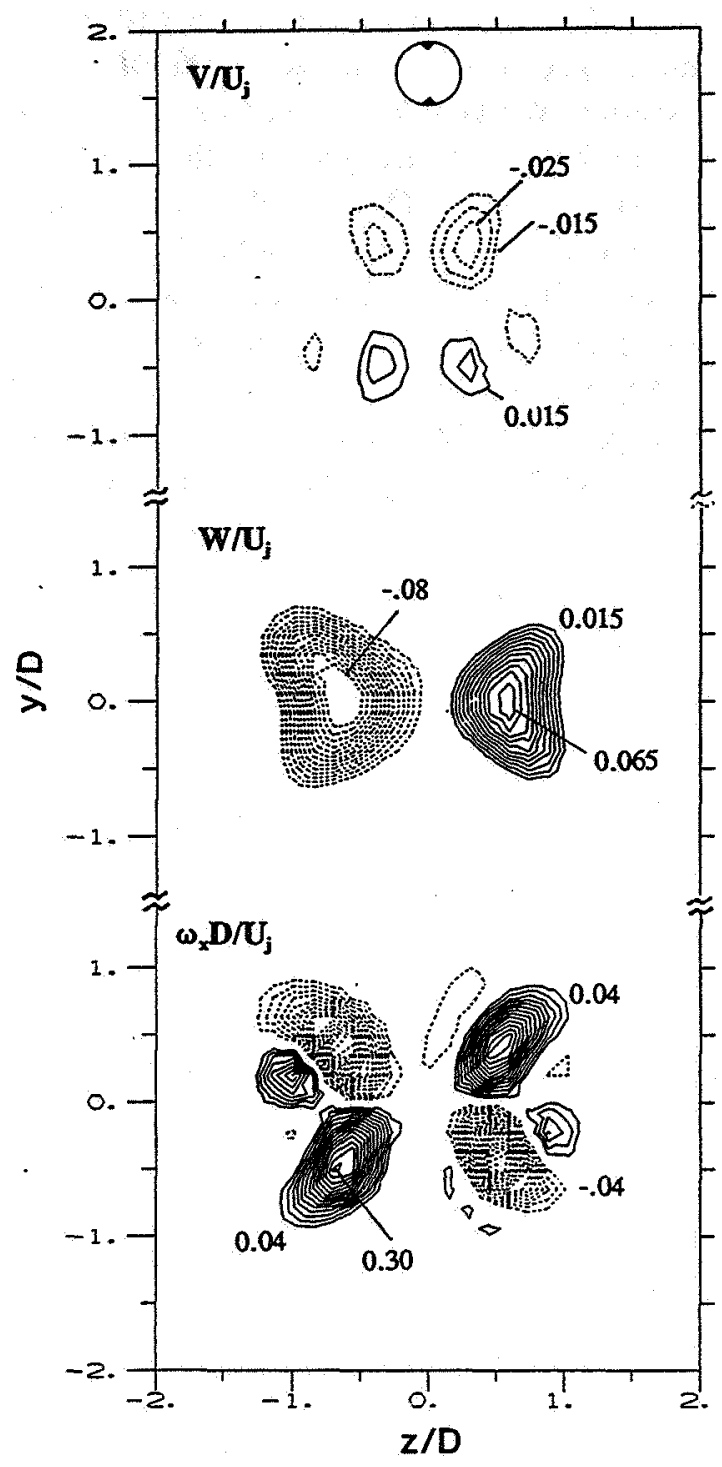

Fig. 12 Contours of indicated quantities for the 2 delta-tabs case; $x / D=3, M_{j}=0.3$.

The distributions of the mean velocities $\mathrm{V}$ and $\mathrm{W}$ at $x / D=3$, for the 2 delta-tabs, are shown by the upper two sets of data in Fig. 12. Corresponding data for the no-tab case yielded values below the minimum contour levels, and thus are not shown. It can be seen that with the specific placement of the delta-tabs, shown schematically on the top of Fig. 12, larger values of $W$ are induced. This is commensurate with the fact that the jet core is mainly spread apart in the z-direction. The 
gradients of $\mathbf{V}$ and $\mathbf{W}$ provided the streamwise vorticity, $\omega_{\mathrm{x}}(=\partial \mathrm{W} / \partial \mathrm{y}-\partial \mathrm{V} / \partial \mathrm{z})$. The corresponding distribution of $\omega_{x}$ are shown at the bottom of Fig. 12. These data unambiguously prove that a pair of streamwise vortices is produced by each delta-tab. The sense of rotation of these vortices, conjectured before, is also confirmed by these data (the solid and dashed contours of $\omega_{x}$ represent anticlockwise and clockwise sense, respectively).

In obtaining the $\omega_{x}$ data, the averaging effect due to the measurement grid size was checked. At a given $\mathrm{x}$, the location of a $\omega_{x}$-maxima was determined from preliminary data. With this location as the center, $V$ and $W$ were measured at the comers of a square grid. Line integration of the velocities provided $\omega_{x}$. A typical set of $\omega_{x}$ obtained in this manner, as a function of the grid size, is shown in Fig. 13. For grid size smaller than a certain value $\omega_{x}$ can be seen to become invariant; of course, for larger grid size there is an averaging effect resulting in smaller amplitudes. The grid invariance of $\omega_{x}$ was determined in this manner for a few stations and conservative grid sizes were used in all measurements.

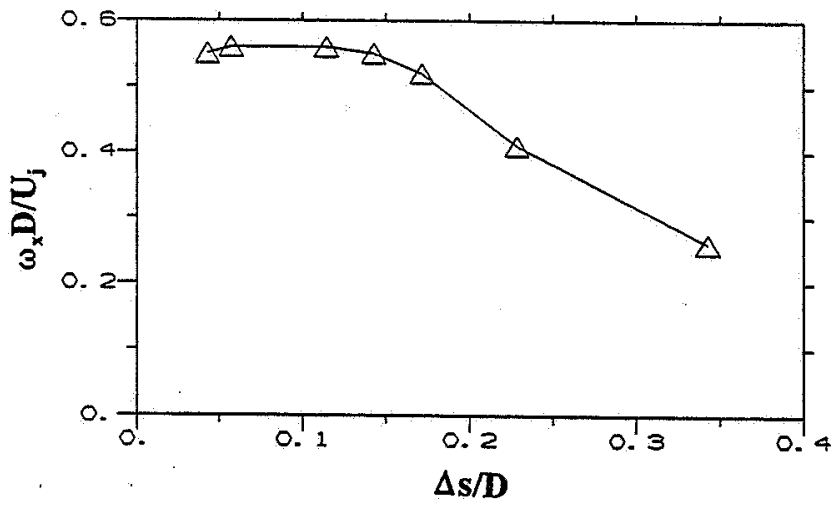

Fig. 13 Effect of spatial resolution on measured vorticity at $x / D=2, y / D=-0.7$ and $z / D=-0.4 ; \Delta s$ is the size of a square measurement grid.

A few more sets of $\omega_{\mathrm{x}}$ data at different $\mathrm{x} / \mathrm{D}$ are presented in Fig. 14. Probe calibration and facility constraints limited the measurements up to $x / D=12$. The vorticity distributions are found to be similar at the upstream stations. With increasing distance the distribution becomes more spread out in the $z$-direction. An
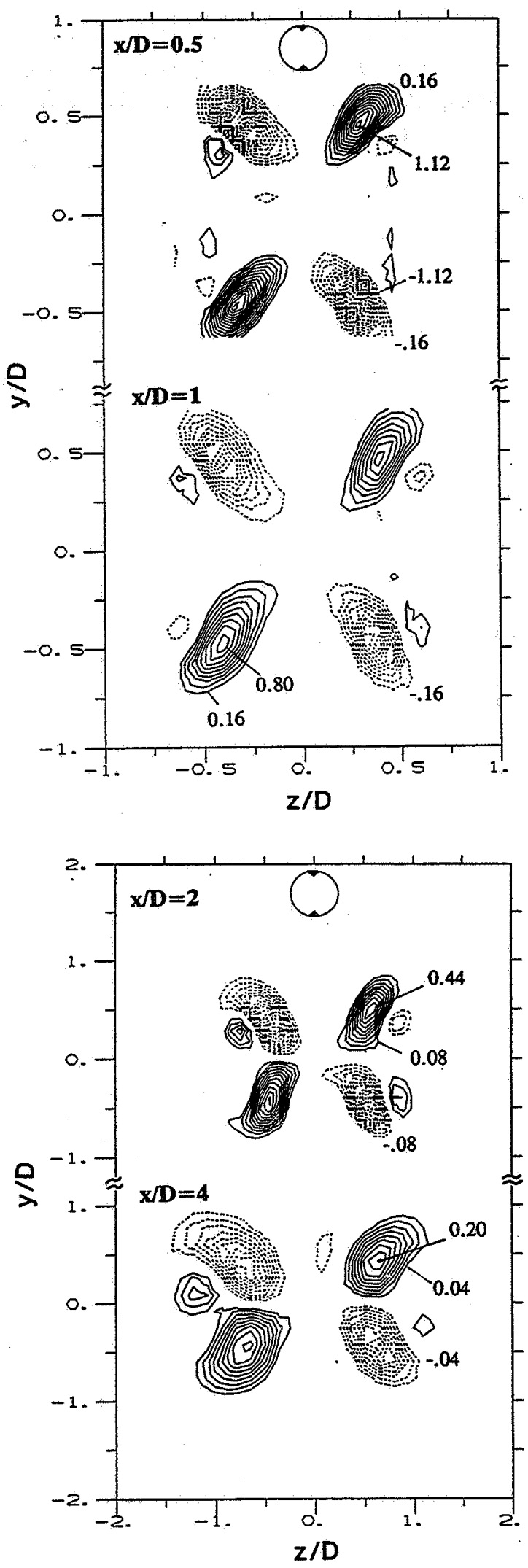

Fig. 14 (Continued) 
inspection of the contour levels shows that the peak amplitudes decay continually with increasing $x / D$. However, the streamwise vortices are clearly detected even at $\mathrm{x} / \mathrm{D}=12$.

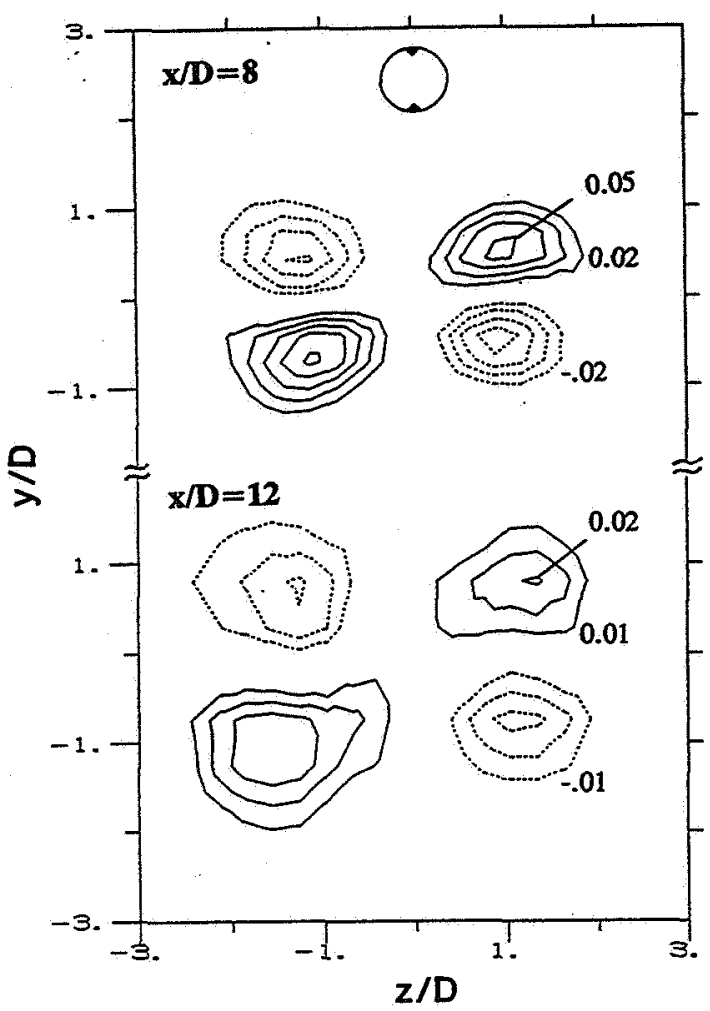

Fig. 14 Contours of streamwise vorticity $\omega_{2} \mathrm{D} / \mathrm{U}_{\mathrm{j}}$ at indicated x/D locations; $M_{j}=\mathbf{0 . 3}$. Contour intervals for $x / D=0.5,1,2,4,8$ and 12 are $0.08,0.08,0.04$, 0.020 .01 and 0.005 , respectively.

The variation of the peak amplitude of $\omega_{x}$ with $x / D$ is shown in Fig. 15. One finds that the strength of the streamwise vortices decay exponentially with $x / D$. However, the variation is continuous and does not show any abrupt decrease anywhere within the measurement range. This suggests that the streamwise vortices apparently have not gone through a 'vortex breakdown' within the measurement range. The issue of vortex breakdown came up in regards to possible mechanisms for the mixing increase by the tabs. The streamwise vortices distort and stretch the mixing layer; the corresponding 'area increase' for the mixing layer surface exposed to the ambient would explain a larger entrainment. Another factor for the larger entrainment could be the breakdown of the streamwise vortices downstream and the resultant increase in turbulence and unsteadiness. The question was the relative role of the two factors in the mixing enhancement. The present results, even though not conclusive, indicate that 'vortex breakdown' may not be a significant factor.

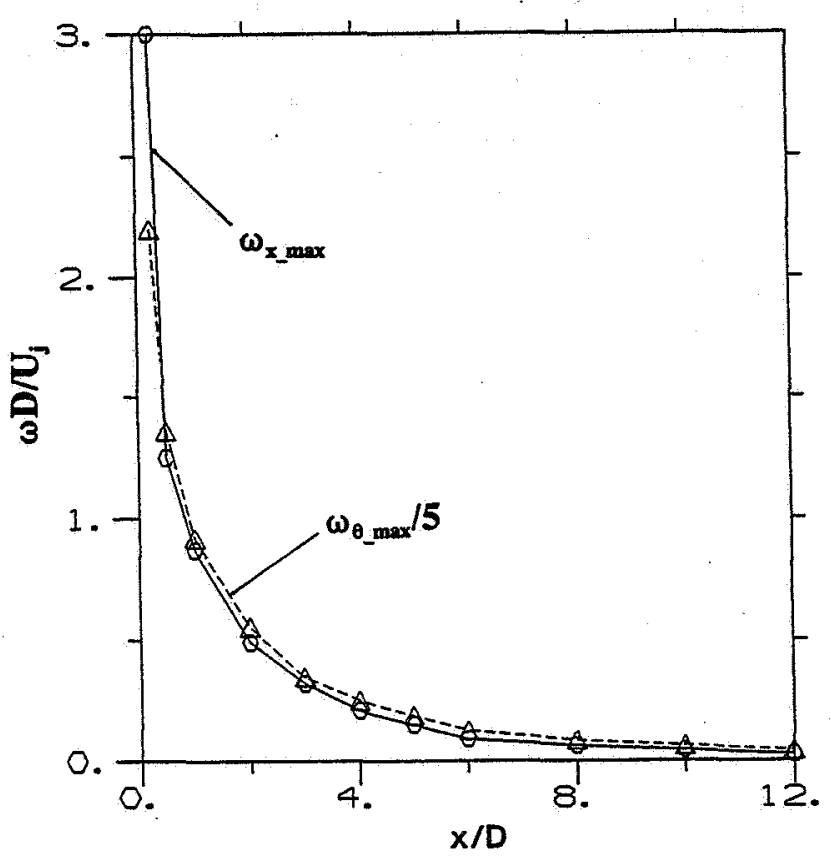

Fig. 15 Variations of maximum streamwise vorticity $\left.\left(\omega_{x},\right)_{1}\right)$ and maximum aximuthal vorticity $\left(\omega_{\theta_{2}}\right)$ with $\mathbf{x} / \mathbf{D} ; M_{j}=0.3$.

Also shown in Fig. 15 is the variation of the peak amplitude of the corresponding azimuthal vorticity $\left(\omega_{\theta)}\right.$. This was approximated from the maxima of $\omega_{y}$ or $\omega_{z}$ at each station. It can be seen that the peak amplitudes of $\omega_{\mathrm{x}}$ and $\omega_{\theta}$ decay simultaneously, the latter component having a magnitude approximately 5 times that of $\omega_{x}$ over the entire measurement range.

3.3 Effect of a delta-tab in a 8:1 rectangular jet: Limited measurements were carried out to compare the effect of one delta-tab in a rectangular jet with the corresponding effect in the axisymmetric jet. The data were taken in the 
smaller jet facility, for $M_{j}=1.63$, with the same deltatab fitted successively to the two nozzles. The total pressure contours measured just downstream of the deltatab are first compared in Fig. 16. The data are qualitative because of measurement error due to shocks in the core of the jet, but should provide a reasonably accurate picture of the defect produced by the tab.

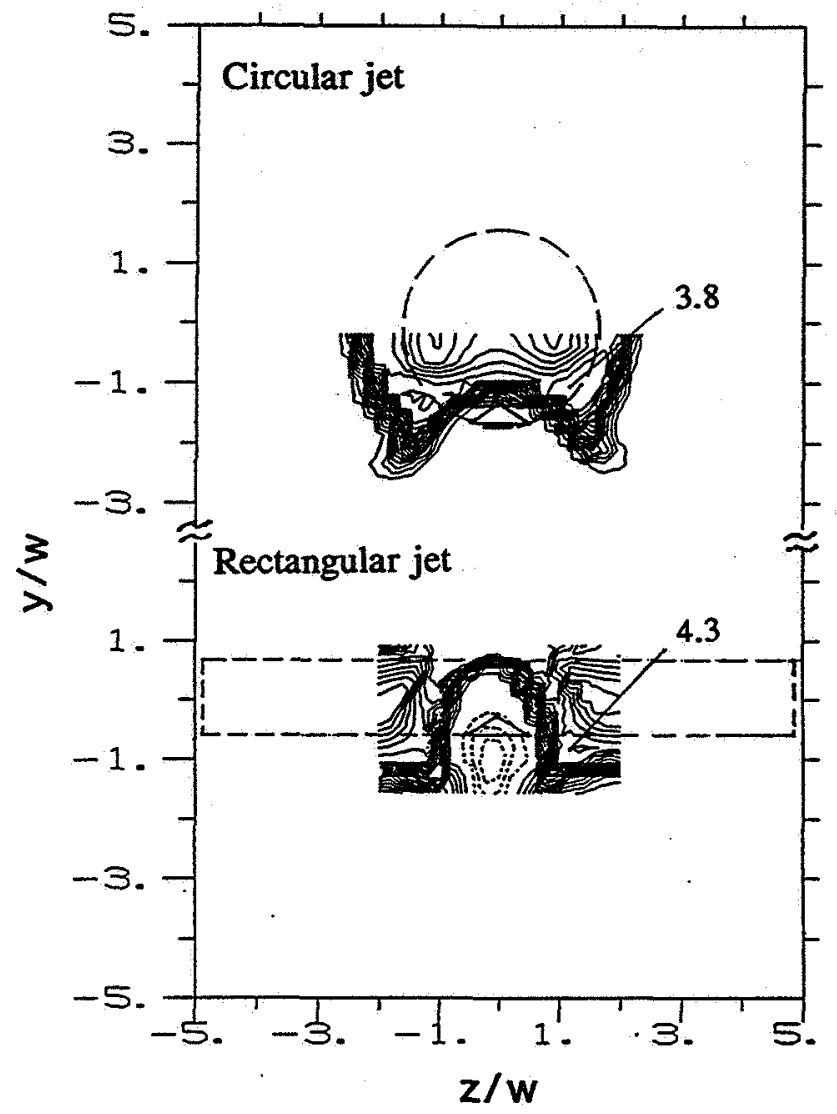

Fig. 16 Total pressure $\left(p_{d} / p_{2}\right)$ contours just downstream of delta-tab $(x / w-0.5) ; M_{j}=1.63$. Diameter of circular jet is $1.27 \mathrm{~cm}$, equivalent diameter of $8: 1$ rectangular jet is $1.47 \mathrm{~cm}$, and $w=0.35 \mathrm{~cm}$ (see Fig. 1).

The co-ordinates $y$ and $z$ in Fig. 16 are normalized by the same quantity ( $w$, see Fig. 1) so that the defect produced by the delta-tab can be directly compared. The outline of the nozzles and the delta-tab are shown by the dashed lines. The darker regions with the concentrated contours represent the mixing layer. The few dashed contours in the middle of the defect for the rectangular case represent subatmospheric pressures read by the Pitot probe. A static pressure probe was later used to read the minimum value of $\left(P_{-}-p_{2}\right) / p_{k}$ at the corresponding locations downstream of the delta-tab. It was read to be about -0.22 for the rectangular case whereas it was practically zero for the axisymmetric case. However, it can be clearly seen that the defect produced is more pronounced in the axisymmetric case. The two corners of the distorted mixing layer on the lower side have spread apart in this case. In contrast these appear to have been drawn closer together in the rectangular case, as if the defect is in the process of being closed up.

Corresponding total pressure contours farther downstream at $x / D=2$ are compared for the two cases in Fig. 17. The evolution of the defect confirm the aforementioned trends. For the axisymmetric case a large indentation has been produced (compare with Fig. 2a).

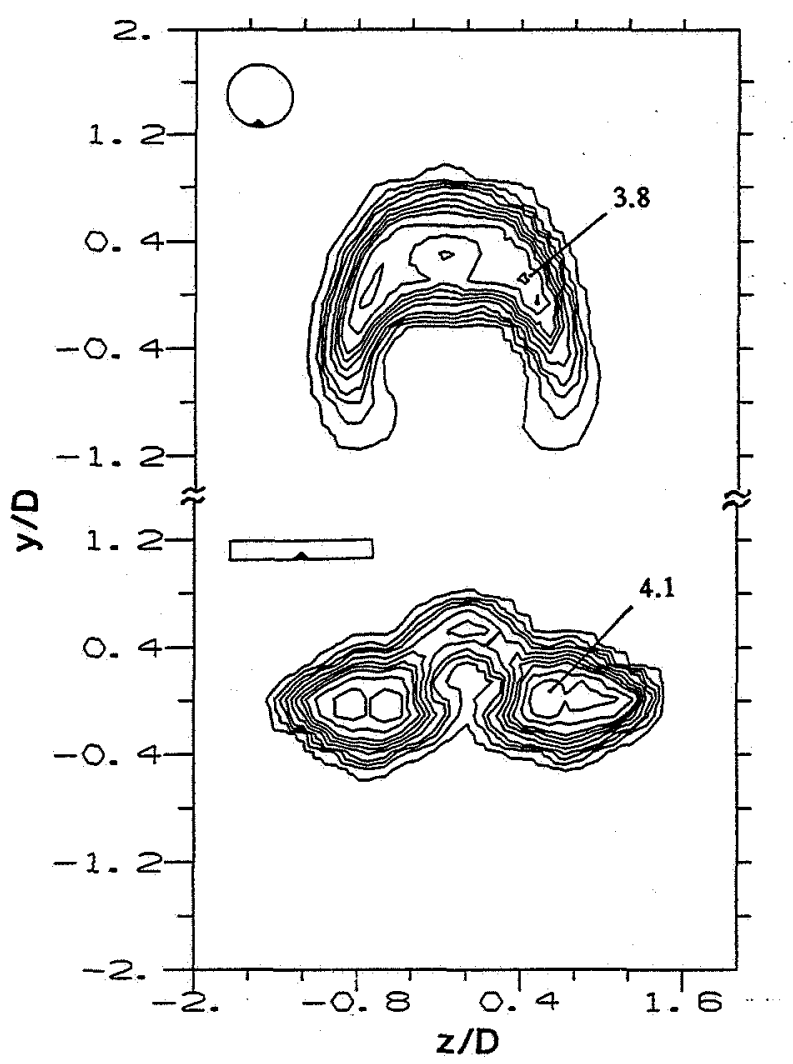

Fig. 17 Total pressure $\left(p_{o} / p_{J}\right)$ contours at $x / D=2$ for the two cases of Fig. 16 . 
But in the rectangular case the indentation is smaller and seemingly disappearing. Thus, the defect produced by the delta-tab appears to be influenced significantly by the upstream flow geometry. It may be conjectured that stronger streamwise vortices are produced in the axisymmetric case resulting in a more pronounced defect.

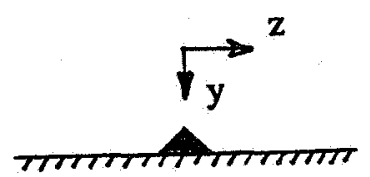

Plane

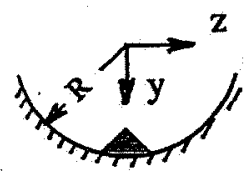

Axisymmetric
Fig. 18 Co-ordinates for plane and axisymmetric configurations.

Equation 1 discussed in section 3.1 applies to a plane configuration and is derived from the z-component of the Navier-Stokes equation by invoking the no-slip condition at the wall. The corresponding equation in cylindrical coordinates can be obtained similarly; Fig. 18 shows the coordinate systems used for this purpose. For the axisymmetric case, replacing the $(y, z)$ coordinates with polar coordinates $(r, \psi)$ where $\psi$ is measured from the $y$-axis, one can obtain Eqn. 2.

$$
\frac{1}{\rho R} \frac{\partial p}{\partial \psi}=v \frac{\partial \omega_{x}}{\partial r}+\frac{\omega_{x}}{R}
$$

This is the corresponding equation for vorticity flux in the axisymmetric geometry under consideration. Note that for $R \rightarrow \infty$, Eqn. 2 reduces to Eqn. 1. Also, for small values of $\Psi$, reverting back to the cartesian $(y, z)$ co-ordinates one finds that the left hand side term and the first term on the right hand side in the two equations are identical. The difference lies in the second term on the right hand side of Eqn. 2.

The maximum pressure generated upstream of the tab may be expected to be approximately the same in the two configurations. The high pressure is generated because the flow is brought to a halt. If the lateral gradient $\partial p / \partial z$ produced in the two cases were also the same, by inspection of Eqns. 1 and 2, one would expect more amount of $\omega_{x}$ produced in the rectangular configuration. However, the results discussed in the foregoing suggest to the contrary. The issue remains inconclusive at the present time and will require further investigation. It appears that the magnitude of $\partial p / \partial z$ is somehow augmented significantly in the axisymmetric geometry which results in a larger net amount of $\omega_{\mathbf{x}}$.

Finally, Fig. 19 is included to show the difference in the jet evolution farther downstream for the two cases of Figs. 16 and 17. These measurements are for $x / D=$ 14, which is far enough downstream so that the flow was everywhere subsonic. The static pressure was also nearly equal to the ambient pressure. Thus, the total pressure data alone provided the corresponding Mach number distribution, which are shown for the two cases. The indentation produced by the delta-tab has practically vanished in both cases. However, in the rectangular case, the jet cross section has actually bulged on the lower side where the indentation was produced by the delta-tab.

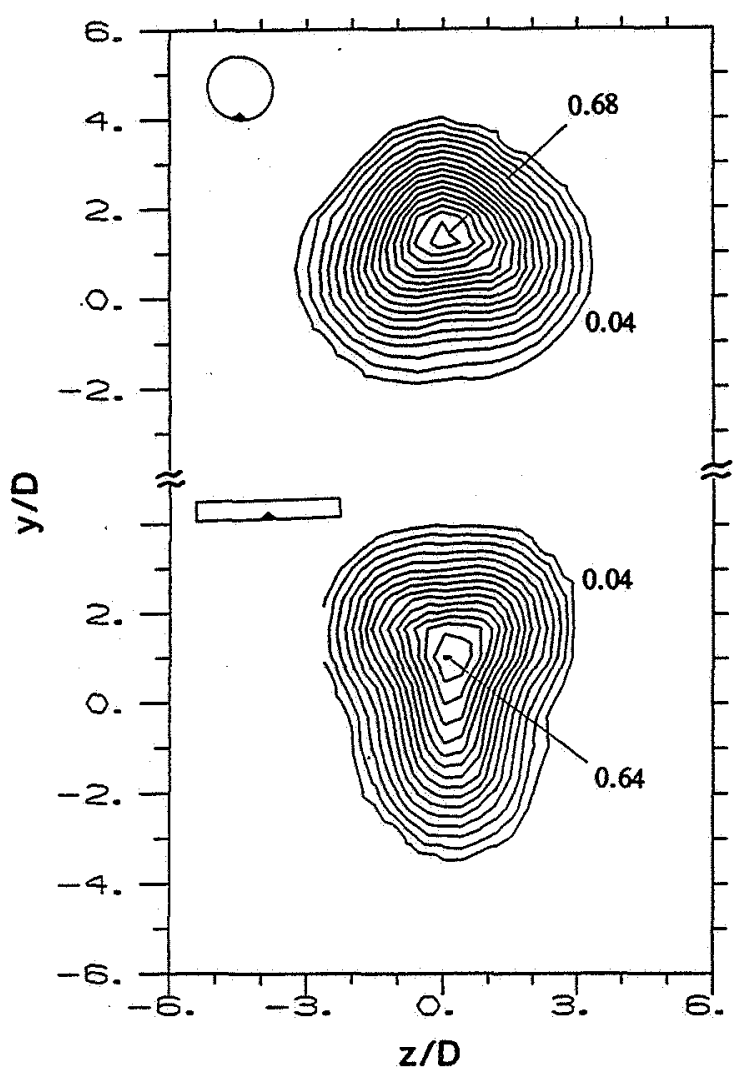

Fig. 19 Mach number contours at $x / D=14$ for the two cases of Figs. 16 and 17. 
The Mach number data were integrated to obtain the mass fluxes. The normalized flux $\mathbf{m} / \mathrm{m}_{e}$, where $\mathrm{m}_{e}$ is the initial mass flux, turned out to be 3.57 and 3.78 for axisymmetric and the rectangular cases, respectively. Thus, the normalized flux for the rectangular case is slightly higher. Corresponding data for the no-tab cases were also obtained (not shown). When the flux values for the tab case are compared with the respective no-tab case, about $25 \%$ increase is observed in the axisymmetric jet. In the rectangular jet, however, a decrease by about $15 \%$ is observed. Thus, these data demonstrate that mixing can also be adversely affected by the tabs, and underscore the need for obtaining a better knowledge of the processes if the tabs are to be used intelligently and profitably.

\section{Conclusions}

A pair of streamwise vortices originate from each delta-tab. These distort the jet cross section dramatically and usually result in more entrainment and mixing as measured downstream. Two sources of the streamwise vorticity are postulated. One is the upstream 'pressure hill' produced by the tab. Another is due to vortex filaments shed from the sides of the tab and reoriented downstream by the mean shear of the mixing layer. In the case of a 'delta-tab', vorticity from the two sources complement each other explaining the stronger effect in that configuration. Source 1 is inferred to be the dominant one in the flow under consideration.

Detailed data on the flow field and vorticity evolution for the effect of two delta-tabs are documented in this paper. The jet decay is much faster under the influence of the delta-tabs. The turbulent stresses are measured to be substantially higher in the tab case. The data confirm that a pair of counter rotating streamwise vortices originate from each delta-tab. The streamwise vortices persist even at the farthest measurement station of $x / D=12$. The magnitude of $\omega_{x}$-maximum is found to decay exponentially with distance from the nozzle, its ratio to azimuthal vorticity $\left(\omega_{\vartheta}\right)$ maximum is found to be about 5 everywhere.

The relative effect of a delta-tab on jets from an axisymmetric nozzle versus a 8:1 rectangular nozzle has been briefly investigated. The distortion produced by the tab is found to be less pronounced in the rectangular case. While mixing and entrainment are increased in the axisymmetric jet, it is actually decreased in the rectangular jet under the influence of one delta-tab. Obviously, this result pertains to the specific tab and nozzle configuration. The effect of the delta-tab(s) when placed at other locations on the periphery of the exit, or in rectangular jets of other aspect ratios, remain to be explored.

\section{References:}

'Samimy M., Zaman K.B.M.Q. \& Reeder M.F., "Effect of tabs at the nozzle lip on the flow and noise field of an axisymmetric jet", ALAA J., 31(4), pp. 609-619, 1993.

'Zaman K.B.M.Q., Reeder M.F., \& Samimy M., "Supersonic Jet Mixing Enhancement by 'Delta-tabs'", Presented at the 28th Joint Prop. Conf., ALAA Paper No. 923548, July 1992.

${ }^{3}$ Zaman K.B.M.Q., Reeder M.F., \& Samimy M., "Control of an axisymmetric jet using vortex generators", Physics of Fluids A (submitted), 1993.

${ }^{4}$ Ahuja, K.K., Manes, J.P., Massey, K.C. \& Calloway, A.B., "An evaluation of various concepts of reducing supersonic jet noise", AlAA Paper No. 90-3982, 1990.

${ }^{5}$ Raman, G., Zaman, K.B.M.Q. \& Rice, E.J., "Initial turbulence effect on jet evolution with and without tonal escitation", Physics of Fluids A, 1(7), 1240-1248, 1989.

${ }^{6}$ Bell J.H. \& Mehta R.D., "Measurements of the streamwise vortical structures in a plane mixing layer" ${ }^{n}, J$. Fluid Mech., 239, 213-248, 1992.

${ }^{7}$ Reeder, M.F. \& Samimy, M., "The evolution of a jet with vortex-generating tabs: visualization and analysis of mean and instantaneous properties"; J. Fluid Mech. (submitted), 1993.

${ }^{8}$ Reynolds W.C. \& Carr L.W., "Review of unsteady, driven, separated flows", ALAA paper 85-0527, 1985.

'Waitz I.A., Marble F.E. \& Zukoski E.E., "Vorticity generation by contoured wall injectors", AlAA Paper 923550, 1992.

${ }^{10}$ Bradshaw, P., "Turbulent secondary flows", Ann. Rev. Fluid Mech., 19, pp. 53-74, 1987.

${ }^{1}$ Potter, M.C. \& Foss, J.F., Fluid Mechanics, Great Lakes Press, Inc. Okemos, Michigan 48864, 1982.

${ }^{12}$ Bridges, J.E. \& Miller, C.J., "Simple tab jet - flow simulation and analysis of vortical mixing mechanisms", Bull. Am. Phys. Soc. vol 37, No. 8, (Division of Fluid Dynamics), 1992.

${ }^{13}$ Ahuja K.K. \& Brown W.H., "Shear flow control by mechanical tabs", ALAA Paper No. 89-0994, 1989.

${ }^{14}$ Bradbury L.J.S. \& Khadem A.H., "The distortion of a Jet by Tabs", J. Fluid Mech., vol. 70, 801-813, 1975.

${ }^{15}$ Crow S.C. \& Champagne F.H., "Orderly structures in jet turbulence", J. Fluid Mech., 48, 547-591, 1971. 
Public reporting burden for this collection of information is estimated to average 1 hour per response, including the time for reviewing instructions, searching existing data sources, gathering and maintaining the data needed, and completing and reviewing the collection of information. Send comments regarding this burden estimate or any other aspect of this Davis Highway, Sulte 1204, Arlington, VA 22202-4302, and to the Office of Management and Budget, Paperwork Reduction Project (0704-0188), Washington, DC 20503.
1. AGENCY USE ONLY (Leave blank)
2. AEPORT DATE
June 1993
3. REPORT TYPE AND DATES COVERED
Technical Memorandum

4. TITLE AND SUBTTILE

Streamwise Vorticity Generation and Mixing Enhancement in Free Jets by

'Delta-Tabs'

6. AUTHOR(S)

WU-505-62-52

K.B.M.Q. Zaman

8. PERFORMING ORGANIZATION REPORT NUMBER

National Aeronautics and Space Administration

Lewis Research Center

Cleveland, Ohio 44135-3191

E-7955

9. SPONSORINGMONITORING AGENCY NAME(S) AND ADDRESS(ES)

10. SPONSORING/MONITORING AGENCY REPORT NUMBER

National Aeronautics and Space Administration

Washington, D.C. 20546-0001

NASA TM-106235

AIAA-93-3253

\section{SUPPLEMENTARY NOTES}

Prepared for the AIAA Shear Flow Conference, sponsored by the American Institute of Aeronautics and Astronautics, Orlando, Florida, July 6-9, 1993. Responsible person, K.B.M.Q. Zaman, (216) 433-5888.

12a. DISTRIBUTION/AVAILABILITY STATEMENT

12b. DISTRIBUTION CODE

Unclassified - Unlimited

Subject Category 02

\section{ABSTRACT (Maximum 200 words)}

The effect of triangular tabs, placed at the nozzle exit, on the evolution of free jets is investigated. The effect, a large distortion of the jet cross section and a resultant increase in mixing downstream, has been inferred before to be due to a pair of streamwise vortices originating from each tab. In this paper, the generation mechanism of the streamwise vorticity $\left(\omega_{x}\right)$ is considered first. Two sources are postulated. One is the upstream 'pressure hill', produced by the tab, which appears to be the dominant source. Another is due to vortex filaments shed from the sides of the tab and reoriented downstream by the mean shear of the mixing layer. In the case of a 'delta-tab', a triangular tab with its apex leaning downstream, vorticity from the two sources add explaining the stronger effect in that configuration. Data on the vorticity evolution for the effect of two delta-tabs are presented, up to twelve jet diameters from the exit, which show that the streamwise vortices persist even at the farthest measurement station. The magnitude of $\omega_{x}-$ maximum decays continually with distance from the nozzle, its ratio to azimuthal vorticity $\left(\omega_{\theta}\right)$ maximum is found to be about $1 / 5$ everywhere. The relative effect of a delta-tab on jets from an axisymmetric nozzle and a 8:1 rectangular nozzle is also studied. The mixing layer distortion is found to be less pronounced in the rectangular case. The jet mixing, as manifested by the mass flux measured at a downstream station, is increased in the axisymmetric jet but it is decreased in the rectangular jet under consideration by the delta-tab.

14. SUBJECT TERMS

Streamwise vortices; Vortex generators; Supersonic jets; Mixing

17. SECURITY CLASSIFICATION OF REPORT

Unclassified
18. SECURTY CLASSIFICATION OF THIS PAGE Unclassified
19. SECURITY CLASSIFICATION OF ABSTRACT Unclassified 